\title{
Equações de Chuvas Intensas Generalizadas para os Estados de São Paulo e Paraná
}

\author{
Leonardo Nitsch Falaguasta Abel Maia Genovez \\ Faculdade de Engenharia Civil-UNICAMP, Depto. de Recursos Hídricos, \\ leonardofala@zipmail.com.br,_genovez@fec.unicamp.br \\ Recebido: 03/02/03 - revisão: 10/06/03 - aceito: 03/07/03
}

\section{RESUMO}

Neste trabalho são analisadas e ajustadas equações de chuvas intensas generalizadas para os estados de São Paulo e Paraná. Foram usadas as séries pluviográficas anuais de 49 postos de chuva para a obtenção dos parâmetros $a_{1}$, b e c, através de processo de otimização, a serem utilizados nos métodos Chen (1983) e Chen (1983) modificado por Hernandez. (1991) denominados respectivamente CHEN NOVO e CHEN AD APTADO NOVO, devido aos novos parâmetros. As curvas dos parâmetros $a_{1}$, b e c foram traçadas a partir do reagrupamento dos valores iniciais de $\mathrm{h}_{1}^{\mathrm{T}} / \mathrm{h}_{24}^{\mathrm{T}}$ com a obtenção de 16 novos valores desta relação. Além dos dois métodos já citados também foram estudados os métodos de Bell (1969) e suas adaptações feitas por Uehara et al. (1980) para o Estado de São Paulo e Brasil, denominados respectivamente BELL ADAPTADO SP e BELL ADAPTADO BR. Foi feito um estudo dos desvios entre as alturas de chuva calculadas com os métodos e as observadas e constatou-se que de maneira geral, ou seja, levando em conta todas as duracões estudadas o método CHEN NOVO apresentou o melhor desempenho.

Palavras-chave: chuvas intensas, pluviógrafo.

\section{INTRODUÇÃO}

Pelo fato de haver em nosso país carência de uma rede de obtenção de dados pluviográficos bem distribuída e com boa confiabilidade, faz-se necessário o emprego de equações que forneçam uma estimativa das alturas máximas de chuva, como as equações de chuvas intensas generalizadas, para projetos de engenharia localizados em pontos sem boas estações ou mesmo sem elas.

É importante salientar que estes métodos não foram desenvolvidos para se adaptar aos mecanismos de formação de chuva orográfica, portanto ficando excluídas da aplicação de qualquer um dos métodos acima citados a região da Serra do Mar e planície costeira no Estado de São Paulo bem como seus respectivos prolongamentos no Estado do Paraná.

\section{Revisão Bibliográfica}

A principal intenção do trabalho desenvolvido por Bell (1969) foi mostrar que o comportamento das chuvas intensas de duração até 2 horas apresenta uma correspondência muito grande em várias regiões do mundo, podendo haver generalização destas. Isto se deve ao fato de que as chuvas de curta duração estão associadas a células de chuva convectivas locais que tem propriedades físicas similares em muitas partes do mundo.

A partir disto Bell (1969) apresentou uma relação altura - duração - freqüência, representada pela equação (1).

$h_{d}^{T}=(0,21 \ln T+0,52)\left(0,54 d^{0,25}-0,50\right) h_{1}^{10}$

Para: $2 \leq \mathrm{T} \leq 100$ anos

$5 \leq \mathrm{d} \leq 120 \mathrm{~min}$

onde:

$\mathrm{h}_{\mathrm{d}}^{\mathrm{T}}$ é a altura de chuva para período de retorno T e duração d em minutos ou horas, $\ln \mathrm{T}$ é o logaritmo natural do período de retorno $\mathrm{T}$ em anos, $\mathrm{d}$ é a duração em minutos e $\mathrm{h}_{1}^{10}$ é a altura de chuva para período de retorno de 10 anos e duração de 1 hora.

A equação (1) permite a estimativa da altura de chuva para qualquer duração de 5 minutos à 2 horas e qualquer período de retorno de 2 à 100 anos, uma vez que seja conhecido o valor da altura de chuva para 10 anos de período de retorno e duração 1 hora $\left(\mathrm{h}_{1}^{10}\right)$.

Em seu estudo, Uehara et al. (1980) seguiram os mesmos passos da metodologia proposta por Bell (1969) ao propor uma relação altura-duração-frequência para todo o Brasil e representada pela equação (2). 


$$
h_{d}^{T}=(0,5800+0,1824 \ln T)\left(0,4966 d^{0,27}-0,5000\right) h_{1}^{10}
$$

Para: $2 \leq \mathrm{T} \leq 100$ anos

$$
5 \leq \mathrm{d} \leq 120 \mathrm{~min}
$$

onde:

$\mathrm{h}_{1}^{10}=$ altura de chuva para 10 anos de período de retorno e duração 1 hora e os demais símbolos iguais aos da equação (1).

No seu estudo, Uehara et al. (1980) também desenvolveram uma relação altura-duração-frequência, representada pela equação (3) apenas para o Estado de São Paulo, desde que seja conhecido o valor da altura de chuva para 10 anos de período de retorno e duração 1 hora $\left(\mathbf{h}_{1}^{10}\right)$ e com os mesmos intervalos de validade citados anteriormente.

$h_{d}^{T}=(0,22 \ln T+0,50)\left(0,38 d^{0,31}-0,39\right) h_{1}^{10}$

Em seu estudo, Chen (1983) desenvolveu uma fórmula generalizada intensidade-duração-frequência de chuva que utiliza três alturas de precipitação, $\mathrm{h}_{1}^{10}$ (chuva de duração de 1 hora e 10 anos de período de retorno), $\mathrm{h}_{24}^{10}$ (chuva de duração 24 horas e 10 anos de período de retorno) e $\mathrm{h}_{1}^{100}$ (chuva de duração 1 hora e 100 anos de período de retorno) e não apenas $\mathrm{h}_{1}^{10}$ utilizada no método de Bell (1969). Ao estudar as relações altura-duração de chuva para durações de até 2 horas, Bell (1969) não levou em consideração as variações da chuva devido às mudanças de localidade geográfica (padrão geográfico da chuva). Estas variações foram consideradas por Chen (1983) na forma da relação $h_{1}^{\mathrm{T}} / \mathrm{h}_{24}^{\mathrm{T}}$ (relação de altura de chuva de duração 1 hora para altura de chuva de duração 24 horas e mesmo período de retorno $\mathrm{T}$ em anos) para qualquer período de retorno e da relação $\mathrm{h}_{\mathrm{d}}^{100} / \mathrm{h}_{\mathrm{d}}^{10}$ (relação de altura de chuva de 100 anos de período de retorno para altura de chuva de 10 anos de período de retorno e mesma duração d em minutos ou horas) para qualquer duração. Ao estudar as relações intensidade-duração $i_{\mathrm{d}}^{\mathrm{T}} / \mathrm{i}_{1}^{\mathrm{T}}$ (intensidade de chuva de duração $\mathrm{d}$ em minutos ou horas para intensidade de chuva de 1 hora e mesmo período de retorno $\mathrm{T}$ em anos), a partir de dados norte-americanos, para durações $\mathrm{d}$ até 24 horas, Chen (1983) verificou que a partir da duração de 2 horas estas relações variavam em função de $\mathrm{h}_{1}^{\mathrm{T}} / \mathrm{h}_{24}^{\mathrm{T}}$. A partir disto, Chen (1983) demonstrou que o método proposto por Bell (1969) não era geral suficiente para responder às variações do padrão geográfico de chuvas, constituindo-se num caso particular da formulação mais geral proposta por ele próprio.

A fórmula generalizada de Chen (1983), desenvolvida para as séries anuais, é dada pela equação (4).

$\mathrm{h}_{\mathrm{d}}^{\mathrm{T}}=\frac{\mathrm{a}_{1} \mathrm{~h}_{1}^{10} \log \left\{10^{(2-\mathrm{x})}\left[\ln \left(\frac{\mathrm{T}}{\mathrm{T}-1}\right)\right]^{-(\mathrm{x}-1)}\right\}}{(\mathrm{d}+\mathrm{b})^{\mathrm{c}}} \times\left(\frac{\mathrm{d}}{60}\right)$

Para: $\mathrm{T} \geq 1$ ano

$5 \min \leq \mathrm{d} \leq 24$ horas.

onde:

$\mathrm{h}_{\mathrm{d}}^{\mathrm{T}}$ é altura de chuva para período de retorno $\mathrm{T}$ em anos e duração d em minutos ou horas, $\mathrm{a}_{1}$, b e c parâmetros de chuva obtidos por Chen (1983) das curvas dos parâmetros de chuva por ele construídas em função de $\mathrm{h}_{1}^{\mathrm{T}} / \mathrm{h}_{24}^{\mathrm{T}}, \mathrm{x}=$ $\mathrm{h}_{1}^{100} / \mathrm{h}_{1}^{10}$, T é o período de retorno em anos, $\mathrm{d}$ é a duração da chuva em minutos e ln é o logarítmo natural.

Considerando os bons resultados obtidos por Chen (1983), Hernandez (1991) propôs uma adaptação daquele método, já que havia a necessidade da obtenção de três alturas de chuva, $\mathrm{h}_{1}^{10}, \mathrm{~h}_{24}^{10}$ e $\mathrm{h}_{1}^{100}$ e no Brasil há grande limitação de dados pluviográficos, particularmente em termos de período de observação, o que torna mais difícil a estimativa de chuvas de curta duração para maiores períodos de retorno como é o caso de $h_{1}^{100}$. Para chuvas de $24 \mathrm{~h}$, podem ser obtidas estimativas melhores face à rede mais densa e os registros muito mais longos dos postos pluviométricos. Com base nestas questões, Hernandez (1991) desenvolveu uma relação altura-duração-frequência baseadas nos seguintes valores de referência: $\mathrm{h}_{1}^{10}$ (chuva de duração 1 hora e 10 anos de período de retorno), $\mathrm{h}_{24}^{10}$ (chuva de duração 24 horas e 10 anos de período de retorno) e $\mathrm{h}_{24}^{100}$ (chuva de duração $24 \mathrm{~h}$ e 100 anos de período de retorno), representada pela equação (5).

$\mathrm{h}_{\mathrm{d}}^{\mathrm{T}}=\frac{\mathrm{a}_{1} \mathrm{D}_{1} \mathrm{~h}_{24}^{10} \log \left[10^{(2-\mathrm{w})} \mathrm{T}^{(w-1)}\right]}{(\mathrm{d}+\mathrm{b})^{\mathrm{c}}} \times\left(\frac{\mathrm{d}}{1440}\right)$

onde: 
$\mathrm{a}_{1}$, b e c são os mesmos parâmetros da equação de Chen, w $=\mathrm{h}_{24}^{100} / \mathrm{h}_{24}^{10}, \mathrm{D}_{1}=24 \times \mathrm{h}_{1}^{10} / \mathrm{h}_{24}^{10}$, d é a duração em minutos e $\mathrm{T}$ é o período de retorno.

A expressão para o parâmetro $\mathrm{D}_{1}$ foi obtida a partir da tabela 3 do trabalho de Chen (1983) onde se apresentam as relações de intensidade de chuva de duração variando de 5 min à $24 \mathrm{~h}$ para intensidade de $1 \mathrm{~h}$ em função da razão $\mathrm{h}_{1}^{\mathrm{T}} / \mathrm{h}_{24}^{\mathrm{T}}$. Como se pode admitir uma relação constante entre chuva máxima de 24 horas e de 1 dia, as alturas de chuva de 24 horas para um determinado período de retorno podem ser substituídas por alturas de chuva com o mesmo período de retorno mas duração de 1 dia, ficando portanto o único dado de pluviógrafo necessário à aplicação do método de Chen (1983) modificado por Hernandez (1991), ou seja, CHEN ADAPTADO o de chuva de período de retorno de 10 anos e duração de 1 hora $\left(\mathbf{h}_{1}^{10}\right)$.

Genovez e Pegoraro (1998) analisaram e avaliaram as equações de chuvas intensas generalizadas propostas por Bell (1969), a de BELL adaptada por Uehara et al. (1980), Chen (1983), a de CHEN adaptada por Hernandez (1991) e as de Chen (1983) e CHEN adaptada por Hernandez (1991) com o uso de novos parâmetros ( $a_{1}$, b e c), obtidos por meio de otimização, a partir dos dados observados de 23 pluviógrafos do Estado de São Paulo. As alturas de chuva calculadas com as equações generalizadas foram comparadas aos valores observados, através do cálculo dos desvios absolutos adimensionais (D) entre os valores calculados e os observados, medidos em relação aos valores observados. De todos os métodos, o que apresentou em média os menores desvios D foi o de Chen (1983) dotado dos novos parâmetros, seguido de perto pelo método de CHEN adaptada por Hernandez (1991), também dotado dos novos parâmetros.

\section{MATERIAL E MÉTODO}

Os postos estudados perfazem um total de 37 no Estado de São Paulo e 12 postos no Paraná, cujas localizações são dadas respectivamente na figura (1). Para o Estado de São Paulo, foram utilizados dados de pluviógrafos fornecidos diretamente pelo Departamento de Águas e Energia Elétrica do Estado de São Paulo (DAEE) e dados de pluviógrafos utilizados por Pegoraro (1996) também de origem do DAEE. Para o Estado do Paraná foram usados dados de parte dos pluviógrafos utilizados por Fendrich (1998), enviados por este autor. Os dados de pluviômetro utilizados neste trabalho, relativos ao Estado de São Paulo, foram obtidos de Pegoraro (1996), já na forma das alturas $\mathrm{h}_{1 \text { dia }}^{10}$ e $\mathrm{h}_{1 \text { dia }}^{100}$, e do Banco de Dados Pluviométricos do DAEE (em CD-ROM). Para o Estado do Paraná, foram obtidos dados de pluviômetro para os postos Ivaiporã e Santa Izabel do Ivaí junto à Superintendência de Desenvolvimento dos Recursos Hídricos e Saneamento Ambiental (SUDERHSA) do Paraná e para os demais postos junto ao Instituto Agronômico do Paraná (IAPAR). Não foram usados postos localizados na Serra do Mar e planícies litorâneas dos dois estados pois as equações estudadas não se adaptam aos mecanismos de chuva orográfica.

Aos 49 postos pluviográficos selecionados dos Estados de São Paulo e Paraná, foi aplicada a distribuição de extremos tipo I (Gumbel) às séries pluviográficas anuais de durações iguais a 10, 20, 30 e 60 min e 2, 3, 6, 12, 18 e $24 \mathrm{~h}$ (os postos do Paraná não possuíam dados de 18h) com a finalidade de se obter os valores das alturas de chuva para os períodos de retorno de 5,10,15, 20, 25, 50 e 100 anos e também às séries pluviométricas de 1 dia para a obtenção das alturas de chuva de períodos de retorno de 10 e 100 anos. Para a estimativa dos parâmetros da distribuição de probabilidades, foi usado o método dos momentos.

Novos parâmetros $a_{1}$, b e c foram estimados a partir das séries pluviográficas para serem usados nos métodos CHEN NOVO e CHEN ADAPTADO NOVO, sendo que a metodologia seguida foi igual à utilizada por Chen (1983). Utilizou-se método de otimização denominado Quasi-Newton cujo algorítmo está descrito em Arcaro (1994).

As alturas de chuva calculadas com as equações generalizadas foram comparadas às alturas observadas por meio do cálculo do desvio relativo expresso em seu valor absoluto (D) entre as alturas calculadas e observadas.

Com os desvios obtidos para todos os postos, foram feitos histogramas onde os métodos puderam ser comparados por duração.

\section{RESULTADOS E DISCUSSÃO}

Com as alturas de chuva para os períodos de retorno desejados obtidas, foram calculadas para os 49 postos as relações de altura de chuva de 1 hora para 24 horas com a obtenção de uma média em porcentagem para os períodos de retorno estudados $\left(\mathrm{h}_{1}^{\mathrm{T}} / \mathrm{h}_{24}^{\mathrm{T}}\right.$ média), sendo obtidos 46 valores desta relação. Para todos os postos também foram calculadas as relações entre intensidade de chuva de duração d e intensidade de 1 hora com a obtenção de uma relação média $\left(\dot{\mathbf{i}}_{\mathrm{d}}^{\mathrm{T}} / \mathbf{i}_{1}^{\mathrm{T}}\right.$ média) para todos os períodos de retorno estudados e para as durações de 10, 20, 30 e 60 min e 2, 3, 6, 12, 18 e 24 horas. Para os postos que apresentaram a mesma relação média $\mathrm{h}_{1}^{\mathrm{T}} / \mathrm{h}_{24}^{\mathrm{T}}$, foram calculadas para cada uma das durações, as médias das relações $\mathrm{i}_{\mathrm{d}}^{\mathrm{T}} / \mathrm{i}_{1}^{\mathrm{T}}$ médias. 


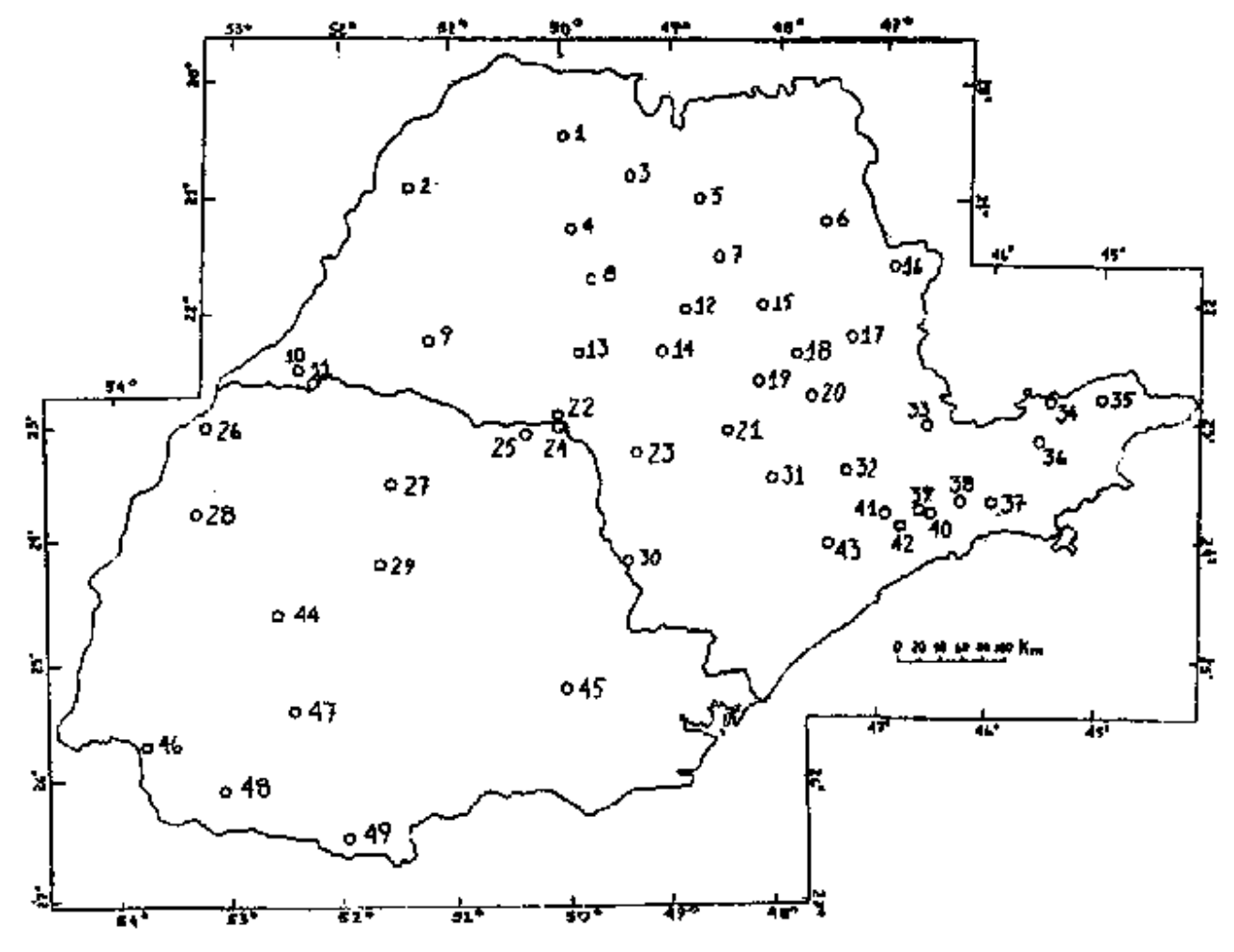
1-B6-036
14- D6-036
27- Apucarana
40- E3-085
2- B8-004
15- C5-017
28- Umuarama
41- E3-034
3- B6-020
16- C3-035
29- Ivaiporã
42- E3-068
4- C6-003
17- D4-030
30- F6-004
43- E4-055
5- C5-043
18- D4-036
31- E5-062
44- Nova Cantu
6- C4-083
19- D5-062
32- E4-023
45- Ponta Grossa
7- C5-106
20- D4-104
33- D3-063
46- Planalto
8- C6-015
21- D5-059
34- D2-096
47- Laranjeiras do Sul
9- D8-041
22- D6-089
35-D2-013
48- Francisco Beltrão
10- D9-005
23- E6-006
36- E2-022
49- Palmas
11- D9020
24- Cambará
37- E2-112
12- C5-055
25- Bandeirantes
38- E3-032
13- D6-092
26- Santa Izabel do Ivaí
39- E3-035

Figura 1 - Área estudada com a localização dos postos pluviográficos. 
A obtenção dos novos parâmetros $\mathrm{a}_{1}$, b e c foi feita aplicando-se os valores da relação $\mathrm{i}_{\mathrm{d}}^{\mathrm{T}} / \mathrm{i}_{1}^{\mathrm{T}}$ média para cada um dos 46 valores de $\mathrm{h}_{1}^{\mathrm{T}} / \mathrm{h}_{24}^{\mathrm{T}}$ média no processo de otimização, sendo que valores iniciais, ou seja, de partida para a otimização foram obtidos em Chen (1983). Com os novos parâmetros obtidos, foram construídos gráficos separados de $\mathrm{a}_{1}$, b e c em função da relação $\mathrm{h}_{1}^{\mathrm{T}} / \mathrm{h}_{24}^{\mathrm{T}}$ média, onde foram ajustadas curvas das quais devem ser extraídos os parâmetros a serem aplicados nos métodos CHEN NOVO e CHEN ADAPTADO NOVO. O problema foi que as curvas ajustadas apresentaram um coeficiente $\mathrm{R}^{2}$ baixo (todos inferiores a 0,9 ). $\mathrm{Na}$ busca de coeficientes $R^{2}$ mais elevados para as curvas, tentou-se uma subdivisão da área estudada em duas sub-regiões onde foram obtidos dois conjuntos de curvas, um para cada sub-região. Mesmo assim algumas curvas ainda apresentaram o $\mathrm{R}^{2}$ inferior à 0,9 . Foi tentado então um terceiro modo para se traçar as curvas dos parâmetros $a_{1}$, b e c. Foi feito um reagrupamento dos 46 valores iniciais de $\mathrm{h}_{1}^{\mathrm{T}} / \mathrm{h}_{24}^{\mathrm{T}}$ média, obtendo-se com isso 16 valores de $\mathrm{h}_{1}^{\mathrm{T}} / \mathrm{h}_{24}^{\mathrm{T}}$ média reagrupada. Novos parâmetros foram conseguidos de forma idêntica à já descrita e foram ajustadas novas curvas desta vez com coeficientes $\mathrm{R}^{2}$ superiores à 0 ,9. Estas curvas com seus coeficientes $R^{2}$, e as suas respectivas equações que permitem a obtenção dos coeficientes $\mathrm{a}_{1}$, b e c a serem aplicados diretamente nos métodos CHEN NOVO e CHEN ADAPTADO NOVO, são ilustradas pelas figuras (2), (3) e (4).

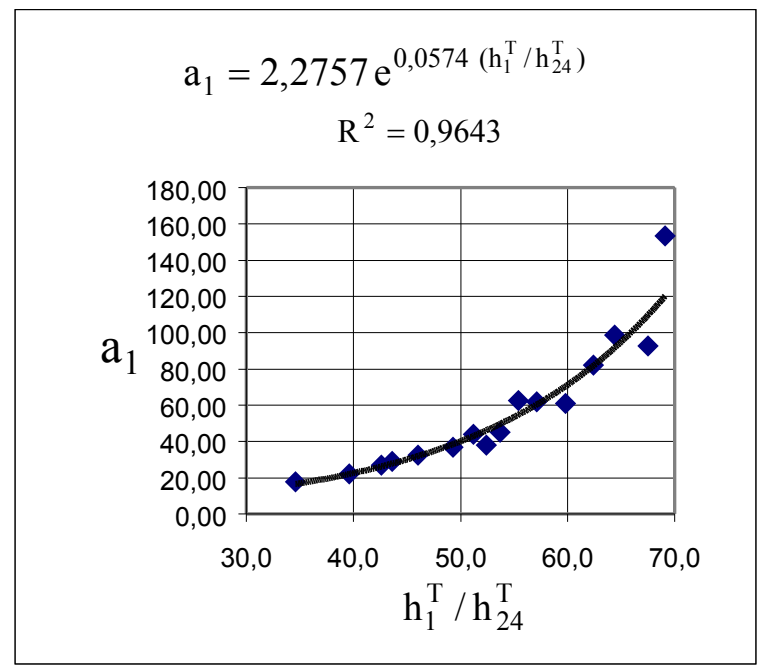

Figura 2 - Curva do parâmetro a construída a partir do reagrupamento.

Foram calculadas as alturas de chuva a partir do método CHEN NOVO, dotado dos novos parâmetros $a_{1}$, b e c obtidos a partir do reagrupamento, do método CHEN ADAPTADO NOVO também dotado destes novos parâmetros, do método de Bell (1969), do método BELL ADAPTADO BR e do método BELL ADAPTADO SP, para as mesmas durações e períodos de retorno das alturas de chuva observadas e para todos os postos distribuídos pela área de estudo.

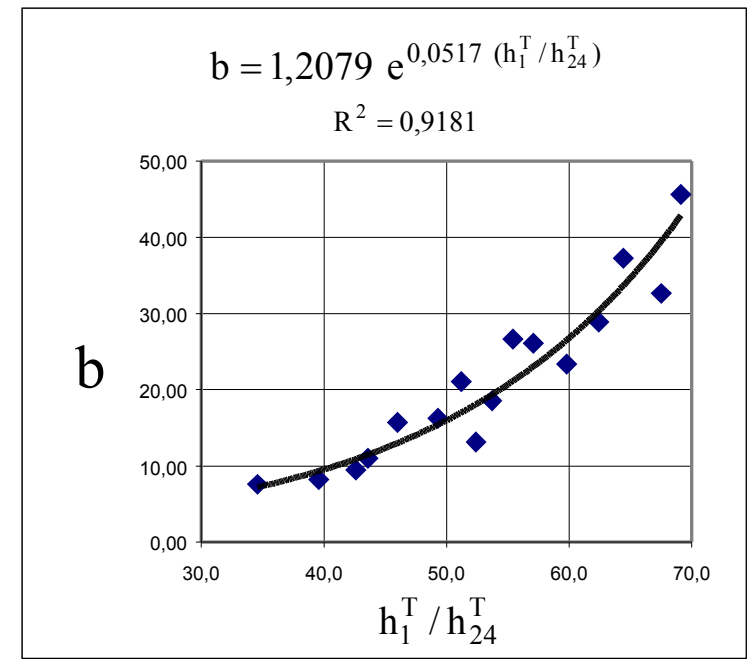

Figura 3 - Curva do parâmetro b construída a partir do reagrupamento.

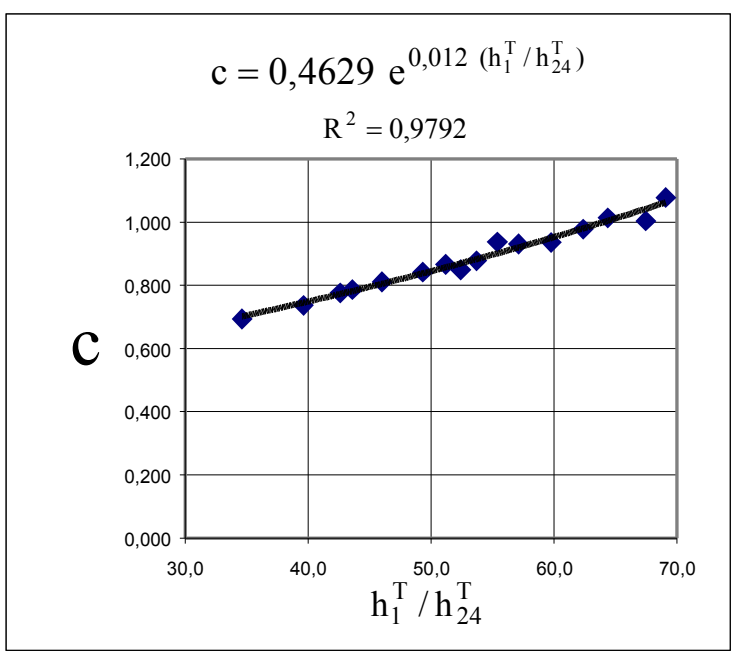

Figura 4 - Curva do parâmetro c construída a partir do reagrupamento.

Foi feito o cálculo dos desvios relativos expressos em seus valores absolutos (D), ou seja, o módulo da diferença entre o valor calculado de altura de chuva e o valor observado de altura de chuva, dividido pelo valor observado de altura de chuva para cada um dos métodos citados anteriormente. 


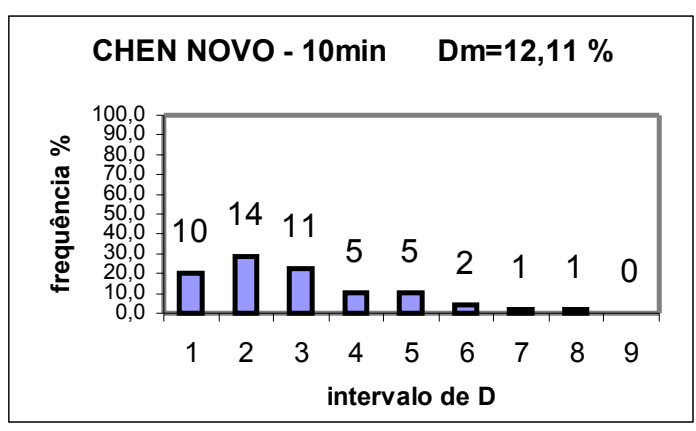

Figura 5

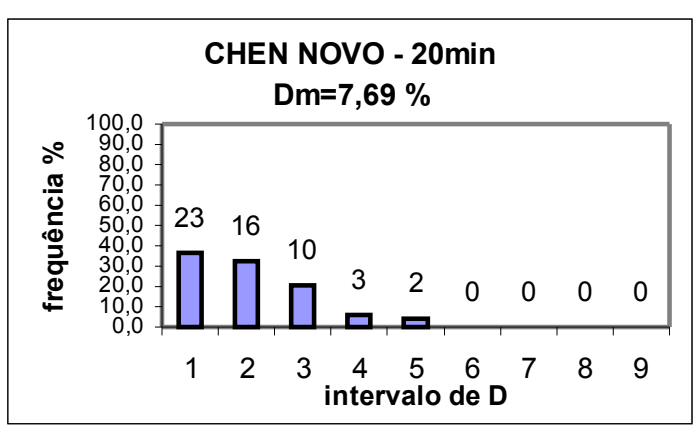

Figura 6

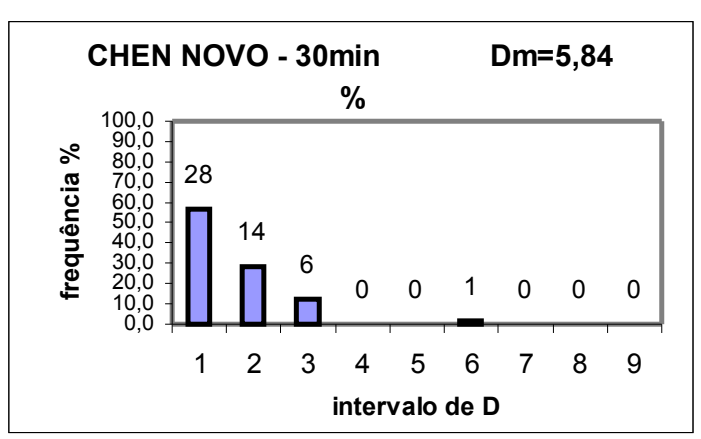

Figura 7

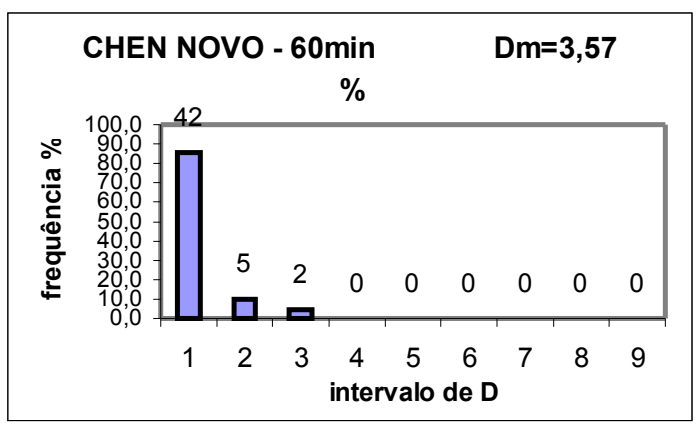

Figura 8

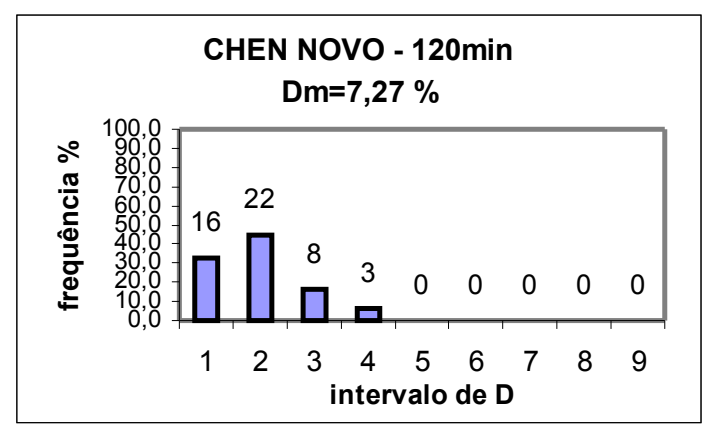

Figura 9

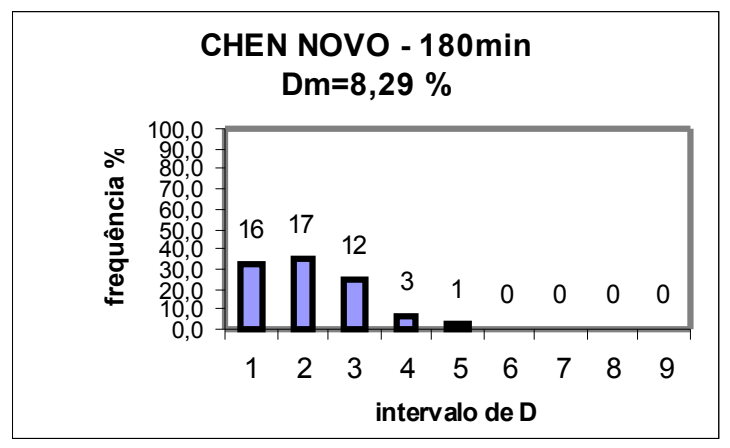

Figura 10

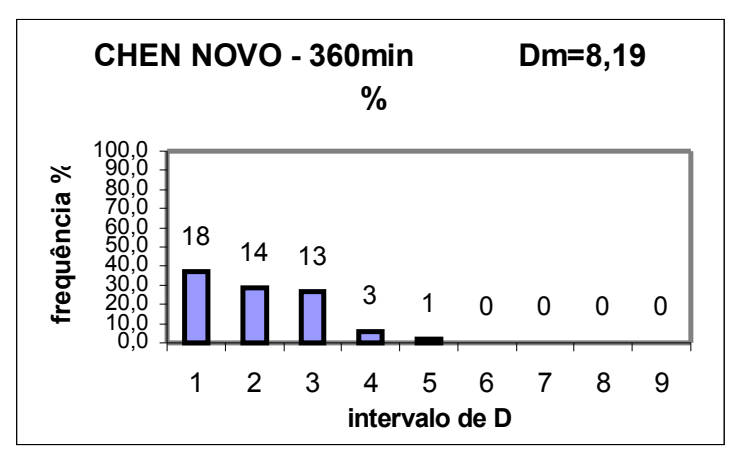

Figura 11

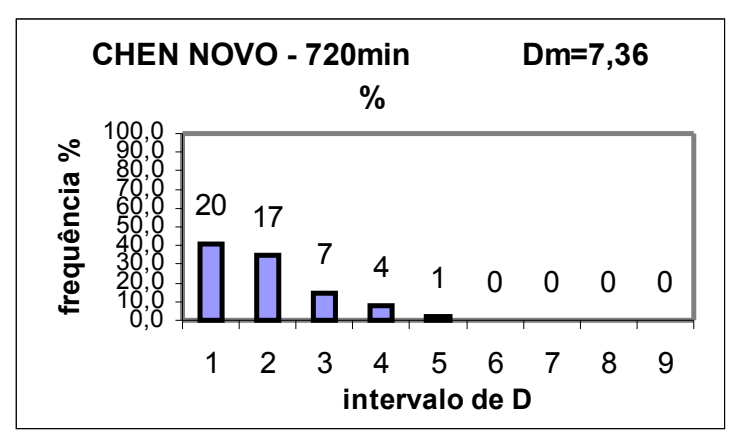

Figura 12 


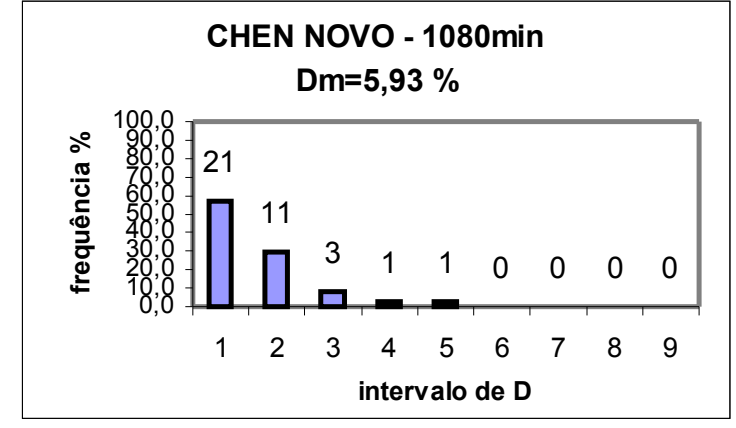

Figura 13

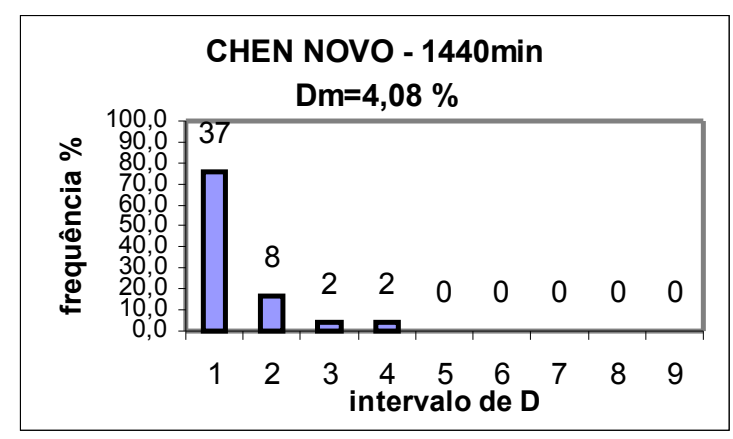

Figura 14

Figuras 5 à 14 - Distribuição da frequência de $\mathrm{D}$ pelos intervalos de $\mathrm{D}$ no método CHEN NOVO para as várias durações estudadas.

A comparação entre os vários métodos foi feita por duração, por meio de histogramas. Na abcissa, cada um dos histogramas apresenta intervalo de $\mathrm{D}$ (desvio relativo expresso em seu valor absoluto) de 1 à 9 , que representam respectivamente $\mathrm{D}$ de 0 à $5 \%, \mathrm{D}$ de $>5 \%$ à $10 \%$, $\mathrm{D}$ de $>10 \%$ à $15 \%, \mathrm{D}$ de $>15 \%$ à $20 \%$, D de $>20 \%$ à $25 \%$, D de $>25 \%$ à 30\%, D de $>30 \%$ à $40 \%, \mathrm{D}$ de $>40 \%$ à $50 \%$ e D de $>50 \%$ à $100 \%$. Na ordenada, cada um dos histogramas apresenta frequência, que é a porcentagem do total de desvios de cada método que cai num determinado intervalo de D. Ainda há sobre todas as barras verticais dos histogramas um valor que corresponde ao número de desvios que caíram naquele intervalo de $\mathrm{D}$, ou seja o valor que deu origem à frequência do intervalo. Cada histograma também apresenta, abaixo de seu título, o valor da média dos desvios relativos expressos em seus valores absolutos (Dm), em porcentagem, correspondente ao método e à duração.

A partir da análise dos histogramas, chegou-se à conclusão que de maneira geral, ou seja, levando-se em conta todas as durações estudadas, o método CHEN NOVO com os novos parâmetros obtidos na otimização e com o reagrupamento foi o que apresentou o melhor desempenho, pois constatou-se que em praticamente todas as durações este método sempre figurou como portador dos melhores indicadores (Dm e distribuição da frequência pelos intervalos de D) entre todos os métodos (os histogramas relativos a este método estão representados nas figuras 5 à 14). Levando-se em conta as durações até 120 minutos, viriam em ordem decrescente após o método CHEN NOVO: o método BELL ADAPTADO SP (que tem sua aplicação restrita ao Estado de São Paulo), BELL ADAPTADO BR, CHEN ADAPTADO NOVO e Bell (1969). Considerando as durações acima de 120 minutos até 1440 minutos CHEN ADAPTADO NOVO teve bom desempenho para 180 minutos, mas se mostrou inferior à CHEN NOVO nas durações superiores. É importante salientar que o método CHEN ADAPTADO NOVO pode ser útil em locais onde se tem carência de dados pluviográficos pois utiliza duas alturas advindas de pluviômetro: $\mathrm{h}_{1 \text { dia }}^{10}$ e $\mathrm{h}_{1 \text { dia }}^{100}$.

\section{CONCLUSÕES}

A realização deste trabalho permitiu a constatação de que: a) as curvas de $\mathrm{a}_{1}$, b e c puderam ser traçadas com maior precisão que as curvas obtidas por Pegoraro (1996), em função da maior quantidade de relações $h_{1}^{T} / h_{24}^{T}$ obtidas; b) para as durações de 10 à 30 minutos os valores da relação $\mathrm{i}_{\mathrm{d}}^{\mathrm{T}} / \mathrm{i}_{1}^{\mathrm{T}}$ obtidos não podem ser considerados independentes da relação $h_{1}^{T} / h_{24}^{T}$ como foi constatado por Chen (1983); c) o método CHEN NOVO com os novos parâmetros obtidos a partir do reagrupamento foi o que apresentou o melhor desempenho.

\section{AGRADECIMENTOS}

Os autores agradecem à CAPES-Fundação Coordenação de Aperfeiçoamento de Pessoal de Nível Superior pela bolsa de mestrado concedida a um dos autores. Agradecem também ao Departamento de Águas e Energia Elétrica do Estado de São Paulo (DAEE-SP), Instituto Agronômico do Paraná (IAPAR), à Superintendência de Desenvolvimento dos Recursos Hídricos e Saneamento Ambiental (SUDERHSA) do Paraná e à Rita de Cássia Fugagnolli Pegoraro pelos dados de chuva fornecidos. Agradecimentos especiais ao Prof. Dr. Roberto Fendrich da PUC-PR pelos dados enviados e ao Prof. Dr. Antônio Carlos Zuffo da FEC-UNICAMP pelo programa de aplicação de distribuição de probabilidades. 


\section{REFERÊNCIAS}

ARCARO, V. F., (1994). Conceitos fundamentais e métodos computacionais básicos para a análise não linear de estruturas. Apostila da Faculdade de Engenharia Civil da Unicamp, 50p.

BELL, F. C., (1969). Generalized rainfall- duration-frequency relationships. Journal of the Hydraulics Division ASCE, vol. 95, No. HY1, p. 311-27.

CHEN, C., (1983). Rainfall intensity-duration- frequency formulas. Journal of Hydraulic Engineering - ASCE, vol.109, No.12, p. 1603-21.

FENDRICH, R., (1998). Chuvas intensas para obras de drenagem no Estado do Paraná. Curitiba, P.R., Ed. Champagnat, $99 \mathrm{p}$.

GENOVEZ, A. M. e PEGORARO, R. C. F., (1998). Análisis y evaluación de ecuaciones decurvas de intensidad generalizadas. In:XVIII Congresso Latinoamericano de Hidráulica - Oaxaca - México, p. 369-378.

HERNANDEZ, V., (1991). Ainda as equações de chuvas intensas - pode-se generalizar? In: IX Simpósio Brasileiro de Recursos Hídricos - Rio de Janeiro - R. J. - Brasil -Anais 3, p. 193-203.

PEGORARO, R. C. F., (1996). Análise e avaliação de equações de chuvas intensas generalizadas. Dissertação de mestrado. Faculdade de Engenharia Civil - UNICAMP - Campinas - S. P. - 99p.

UEHARA, K.; ZAHED FILHO, K.; SILVEIRA, L. N. L.; EIGER, S. E PALERMO, M. A. (1980). Pequenas bacias hidrográficas do Estado de São Paulo - Estudo de vazões médias e máximas. FDTE/Escola Politécnica da USP, São Paulo, S. P., vol.2, 708p.

\section{Generalized Rainfall-Duration-Frequency Rela- tionships to São Paulo and Paraná States - Brazil}

\section{ABSTRACT}

In this work, the annual maximum series of 49 recording gauging stations located in the states of São Paulo and Paraná, Brazil, have been used to obtain parameters $a_{1} b$ and $c$, by mean of an optimization process to be employed in the Chen (1983) method, with and without the modifications proposed by Hernandez. (1991). Besides those two, the methods proposed by Bell (1969) and Uehara et al. (1980) have been studied for the State of São Paulo and Brazil. It has been found that, in general terms and for all durations, Chen's method (1983) with new parameters presented the best performance.

Key-words: intensity rainfall; raingange. 